\title{
A Design Methodology for Compositional High-Level Synthesis of Communication-Centric SoCs
}

\author{
Giuseppe Di Guglielmo, Christian Pilato, Luca P. Carloni \\ Dept. of Computer Science - Columbia University, New York, NY - USA \\ \{giuseppe,pilato,luca\}@cs.columbia.edu
}

\begin{abstract}
Systems-on-chip are increasingly designed at the system level by combining synthesizable IP components that operate concurrently while interacting through communication channels. CAD-tool vendors support this System-Level Design approach with high-level synthesis tools and libraries of interface primitives implementing the communication protocols. These interfaces absorb timing differences in the hardware-component implementations, thus enabling compositional design. However, they introduce also new challenges in terms of functional correctness and performance optimization. We propose a methodology that combines performance analysis and optimization algorithms to automatically address the issues that SoC designers may accidentally introduce when assembling components that are specified at the system level.
\end{abstract}

\section{Categories and Subject Descriptors}

B.5 [RTL Implementation]: Design Aids

General Terms

Algorithms, Design, Experimentation

Keywords

High-Level Synthesis, SystemC

\section{INTRODUCTION}

The complexity of modern Systems-on-Chip (SoC) is driving the adoption of Electronic System Level (ESL) design methodologies that raise the level of abstraction above RTL design and promote the reuse of components [13]. These components are increasingly specified with high-level programming languages, such as $\mathrm{C}++$ and SystemC, to speed up system-level simulation and enable their implementation as energy-efficient hardware accelerators [18].

Fig. 1 shows an abstraction of the hardware part of a typical ESL design flow. An application (or part of an application) that will be implemented in hardware is specified as a set of components which operate concurrently while interacting through communication channels. These components are expressed, for instance, as synthesizable SystemC processes. Their specifications may be the result of partitioning and refinement steps from a higher-level algorithmic description or may be taken from libraries of pre-designed Intellectual Property (IP) blocks. High-level synthesis (HLS) tools are used to synthesize a hardware implementation for each component after performing various micro-architectural optimizations. Indeed, given a SystemC process, SoC designers can obtain several alternative implementations by applying a variety of "HLS knobs" such as: loop unrolling, loop pipelining, resource sharing, etc. These implementations form a Pareto-optimal set of choices in a multi-objective optimization space that represent design tradeoffs in terms of performance metrics versus area/power costs.

Permission to make digital or hard copies of all or part of this work for personal or classroom use is granted without fee provided that copies are not made or distributed for profit or commercial advantage and that copies bear this notice and the full citation on the first page. Copyrights for components of this work owned by others than ACM must be honored. Abstracting with credit is permitted. To copy otherwise, or republish, to post on servers or to redistribute to lists, requires prior specific permission and/or a fee. Request permissions from Permissions@acm.org.

DAC '14, June 01 - 05 2014, San Francisco, CA, USA.

Copyright 2014 ACM 978-1-4503-2730-5/14/06 ...\$15.00.

http://dx.doi.org/10.1145/2593069.2593071.

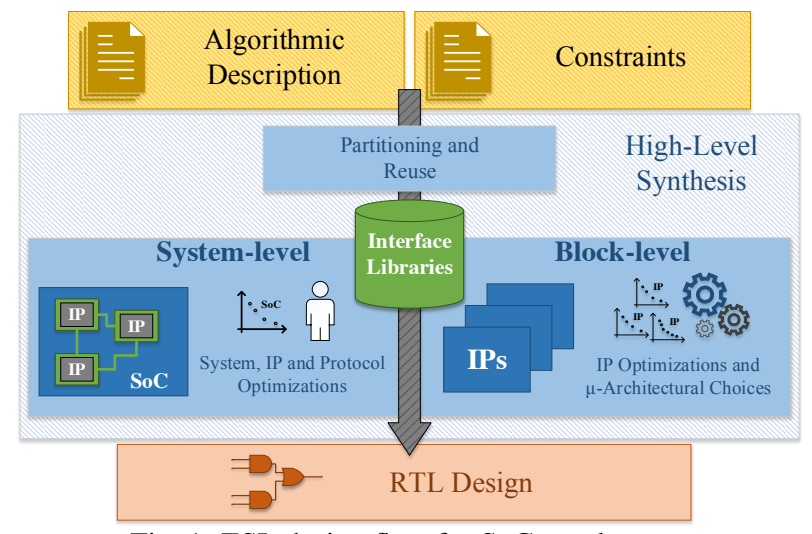

Fig. 1: ESL design flow for SoC accelerators.

State-of-the-art HLS tools offer excellent intra-process optimization but very limited support for inter-process optimization. Hence, choosing the best implementation of each component for a given SoC and combining these implementations into an optimal system design are still manual, time-consuming tasks. To assist SoC designers in this effort, CAD-tool vendors provide libraries of interface primitives. These offer an application programming interface (API) to specify communication and synchronization among processes at the system-level as well as synthesizable implementations that can be combined with the implementation of each process in a modular fashion $[4,7,16]$. Examples of these primitives are the blocking read/write commands to receive/send messages in a synchronized way on a point-to-point channel between two processes. By encapsulating low-level signals, absorbing the timing differences across processes, and providing pre-designed implementations, these interface libraries support Transaction-Level Modeling (TLM) [8] and relieve SoC designers from the tedious task of creating a communication protocol.

On the other hand, the lack of automated tools for performance analysis and optimization at the system level makes the use of these libraries very challenging. The larger the number of components the harder the challenge. In particular, since the interface primitives are called within separate SystemC processes, they introduce serialization into the data transfers across the corresponding components. This may cause performance loss and, in the worst case, system deadlock. We address this problem by making three contributions: (1) a formal model to capture the impact of the interface primitives on system-level performance; (2) an efficient algorithm that, for a given system design, optimizes the use of these primitives in each process while ensuring absence of deadlock; and (3) a CAD tool to optimize the implementation of the computation part within each process and the inter-process communication channels.

Combined these contributions support a novel design methodology that enables compositional HLS and efficient system-level design-space exploration, thus improving ESL design productivity.

\section{MOTIVATING EXAMPLE}

The graph of Fig. 2(a) models a simple system consisting of five processes (represented by vertices $P_{2} \ldots P_{6}$ ) communicating via eight point-to-point unidirectional channels (represented by arcs 

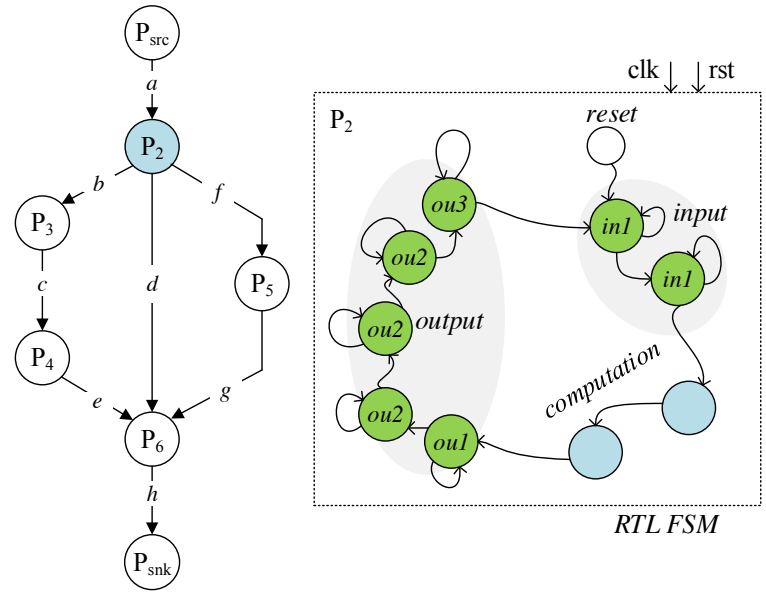

a.

Fig. 2: A motivating example.

$a \ldots h$.) When specifying this kind of systems, designers develop also a testbench that captures the environment in which they operate: this is modeled by vertices $P_{s r c}$ and $P_{s n k}$, which produce and consume data for the system under design, respectively.

A portion of the synthesizable SystemC code of process $P_{2}$ is reported in Listing 1 . The process behavior is specified in an untimed or loosely-timed TLM style [15] following a common structure: after a reset phase (lines 13-14), there is the main infinite loop (lines 15-27). Each loop iteration consists of three phases: input reading (lines 16-18), computation (line 20, hereby omitted), and output writing (lines 22-26). In particular, during the input and output phases the process communicates with other processes using the interface primitives. With the get primitive it acquires input data that are stored in a local memory array (lines 16-18). With the put primitives it emits output data on channels $b, d$ and $f$, respectively. The process executes continuously the sequence of three phases unless suspended due to either an explicit wait () statement in the computation phase or implicit waits present in the implementation of the interface primitives, as explained next.

The interface primitives used in this example implement a blocking communication protocol similar to the one described in [4]: a put in a process (e.g. ou1.put in $P_{2}$, line 23) is associated to a corresponding get in the communicating process at the other hand of a channel (e.g. a get in $P_{3}$ on channel $b$ ); only when both processes reach the corresponding statements the transfer of data can occur. Conversely, if a process reaches its statement before the other, it gets suspended until the other is also ready to communicate. The use of such blocking protocol is very common and all CAD vendors support it by providing API and implementation libraries for similar interface primitives ${ }^{1}$ Notice that the logic to suspend the protocol is transparent to the user as it is specified within the implementation of the get and put primitives.

The use of predefined interfaces simplifies the compositional design and synthesis of SoCs but it is not risk free. Although EDA vendors guarantee the timing and functional correctness of the interface implementations, designers may accidentally introduce system-level bugs or performance degradation when assembling components through these primitives. Debugging these problems is time consuming as it may requires many simulations and repeated HLS tool runs.

The serial nature of the SystemC process induces an order not only among the three main phases of its behavior (input, computation, and output) but also on the execution of the primitive statements. For instance, process $P_{2}$ first sends data to $P_{3}$ on channel $b$ with ou1. put, then to $P_{6}$ on $d$ with ou 2 . put, and finally to $P_{5}$ on $f$ with ou3.put. Similar orders are defined among multiple get

\footnotetext{
${ }^{1}$ Other protocols are also provided. In this paper we focus on blocking protocols but our approach applies also to non-blocking protocols as described in [6]
}

Listing 1: Synthesizable SystemC code of process $P_{2}$.

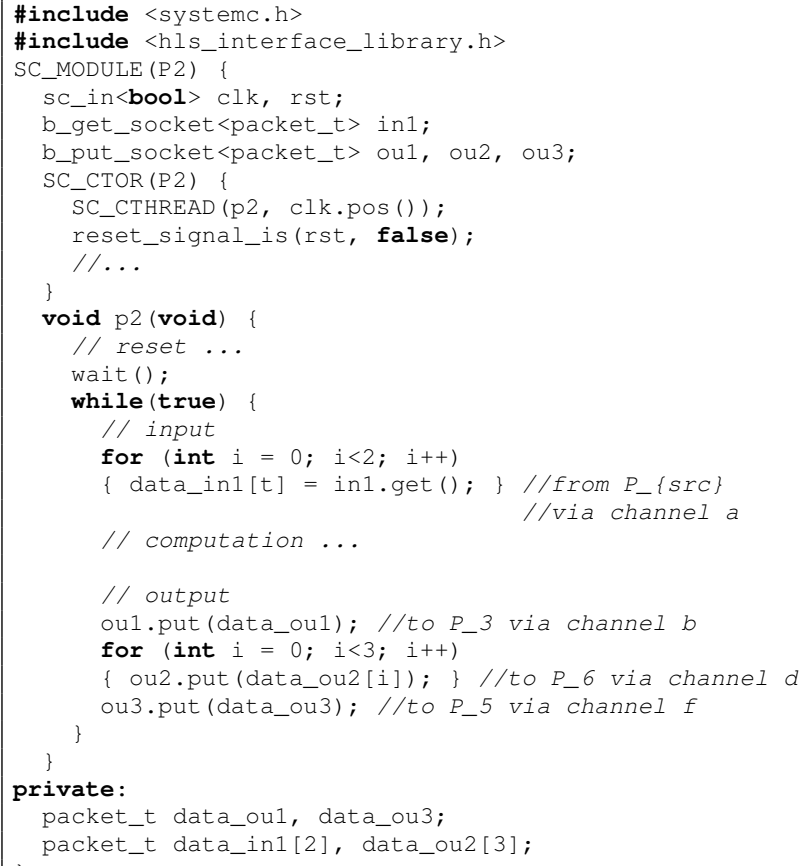

statements. For instance, we could assume that the code of process $P_{6}$ was specified such that it first reads data from $P_{5}$, then from $P_{2}$, and finally from $P_{4}$. If this was the case, however, the combination of these specifications would lead to a major problem: deadlock! In fact, process $P_{2}$ is prevented from sending data to $P_{5}$ on channel $f$ because it is suspended on its ou2 . put on channel $d$ waiting to communicate with $P_{6}$. But $P_{6}$ is suspended on its get on channel $g$ waiting to read from $P_{5}$, which itself cannot reach the put statements in the output phase because is suspended on its get on channel $f$ waiting for $P_{2}$.

To avoid deadlock is necessary to properly reorder the interfaceprimitive statements within the processes. For instance, we could reorder the put statements of $P_{2}$ such that channel $f$ is written before channel $b$, which in turn is written before channel $d$ and, at the same time, reorder the get statements of $P_{6}$ such that channel $e$ is read before $g$, which is read before $d$. But the task of detecting and removing deadlock by code inspection becomes much harder with the complexity of the design. In the simple example of Fig. 2(a) there are already 36 possible order combinations. More generally, this number grows as: $\prod_{p \in \mathcal{P}}((\mid$ in_chan $(p) \mid) ! \times(\mid$ out_chan $(p) \mid) !)$, where $\mathrm{P}$ is the set of processes.

Further, not all deadlock-avoiding orders are the same in terms of system performance: e.g. the second order given above avoids deadlock but yields a design with a suboptimal data-processing throughput because it forces a serial execution among processes that could run concurrently. One may think that such serialization would only impact the simulation speed and not the final implementation because, after all, hardware is inherently parallel. But this is not the case. The serial nature of the SystemC process in combination with the use of interface libraries and HLS may impact negatively the performance of the final hardware implementation.

Example. Consider the finite state machine (FSM) of Fig. 2(b) that is generated by a commercial HLS tool as part of the synthesis of the RTL implementation (Verilog code) for process $P_{2}$ of Listing 1 . This FSM is the result of combining the implementation of the blocking primitives for the I/O phases of $P_{2}$ from the interface library (where the protocol is already implemented in a cycle-accurate manner) with the HLS of the computation phase. The resulting hardware circuit also iterates among the input, com- 
putation, and output phases. There are as many I/O states as the number of get/put statements to be executed in the original process. The self-loop in each of these state allows the circuit to stall for multiple clock cycles when it must wait on a given channel for the circuit implementing the corresponding process to be ready for a data transfer. The chains of input and output states are separated by a chain of computation states. The length of this chain depends on the micro-architecture obtained through HLS: the more parallel is the micro-architecture, the shorter is the chain of computation states (but, generally, the more costly is the circuit implementation in terms of area and power.) A similar FSM is generated for each of the processes of Fig. 2(a). While each FSM enforces a serial progress of the computation of its circuit, naturally all hardware circuits can progress in parallel. Still, the degree of parallelism in the operation of the RTL implementation corresponds directly to the degree of concurrency in the execution of the corresponding SystemC specification. At the core of this correspondence there is the relative ordering of the $\mathrm{I} / \mathrm{O}$ primitive statements. In particular, a shrewd order reduces the number of clock cycles that a component circuit spends waiting for a successful communication. This order, however, depends also on the micro-architectural choices made during the HLS synthesis of the computation part of each process.

Hence, in addition to the question of how to avoid deadlock efficiently, there is a second question: is it possible to jointly optimize the intra-process computations and the inter-process communications in order to maximize the system performance? The rest of the paper will answer positively to both questions.

\section{PERFORMANCE ANALYSIS}

The previous section showed how the main infinite loop in the synthesizable SystemC process $P_{2}$ translates into the cyclic-structure FSM of Fig 2(b), which controls the hardware circuit obtained from $P_{2}$ through HLS. A similar FSM is synthesized as part of the implementation of each other process in the system. Since pair of processes communicate via a blocking interface, the implementation of the channel connecting the corresponding pair of circuits translates also in a cyclic structure, with request/acknowledge signals controlling the synchronized data transfers. In summary, the resulting hardware implementation of the overall system is characterized by a set of cyclic control structures, which have various lengths and interact in a way that is determined by the topology of the system specification. The performance of such deterministic concurrent system can be formally modeled using Timed Marked Graphs. This is a sub-class of Petri Nets can efficiently model concurrent systems, particularly those with periodic behavior [3, 14].

Definition 1. A timed marked graph is a graph defined as a 5tuple $\mathcal{G}=\left(P, T, F, d, M_{0}\right)$, where $P$ is a finite set of places, $T$ is a finite set of transitions, $F \subseteq(P \times T) \cup(T \times P)$ is a set of arcs, $d: T \rightarrow \mathbb{N}^{+}$is a timing function, and $M_{0}: P \rightarrow \mathbb{N}^{+}$is the initial marking, and such that $P \cap T=\varnothing \wedge P \cup T=\varnothing$ and $\forall p \in P .(|\{t .(t, p) \in F\}|=|t .(p, t) \in F|=1)$.

In other words, a TMG is a bipartite directed graph with two kinds of vertices (places and transitions), where each place has exactly one incoming edge and one outgoing edge; the timing function associates a delay to each transition; places can hold zero or more tokens; transitions cannot hold tokens, but they can fire; the initial marking specifies how many tokens each place holds before any firing. A firing creates a new marking by moving tokens around in the graph. A transition is enabled to fire when the place on each of its incoming edges has at least one token. When a transition fires, it takes a token from each of its incoming places and puts a new token into each of its outgoing places. While the firing activity may change the overall number of tokens in a TMG $\mathcal{G}$, the number $M_{0}(c)$ of tokens that are present on a cycle $c$ of $\mathcal{G}$ is invariant under any firing sequence.

Definition 2. The cycle time $\pi(t)$ of a transition $t$ of a TMG $\mathcal{G}$ is the average time separation between two consecutive firings of $t$ and its reciprocal gives the average firing rate of $t$.

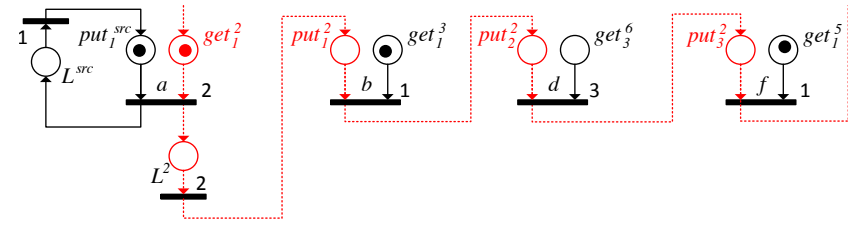

Fig. 3: The TMG model of $P_{2}$ in Listing 1 and Fig. 2.

If $\mathcal{G}$ is strongly connected, then a firing sequence eventually leads $\mathcal{G}$ back to the initial marking $M_{0}$ after firing every transition an equal number of times. All transitions of a strongly-connected TMG $\mathcal{G}$ have the same cycle time, which is called the cycle time of $\mathcal{G}$ and denoted as $\pi(\mathcal{G})$. This is a natural performance metric for the system modeled by $\mathcal{G}$ because its reciprocal is the rate of consumption/production of tokens, i.e., the throughput of the system.

Definition 3. The cycle mean of cycle $c$ of TMG $\mathcal{G}$ is the ratio of the number $M_{0}(c)$ of tokens that are present on $c$ divided by the sum of the delays of its transitions, i.e., $\mu(c)=\frac{M_{0}(c)}{\sum_{t \in c} d(t)}$.

The cycle time $\pi(G)$ of $\mathcal{G}$ is equal to the reciprocal of the minimum cycle mean across all cycles in $\mathcal{G}$. A cycle $c$ with $\mu(c)=$ $\pi(\mathcal{G})$ is critical cycles. Calculating the minimal cycle mean and identifying the critical cycles by the Definition 3 is impractical, since it requires the enumeration of all the elementary cycles of $\mathcal{G}$. More efficient methods exist, based on linear programming [12] or graph theory [5]. For our purposes we adopted Howard's algorithm [2], a polynomial-time algorithm in the size of the problem that is well-known to the stochastic-control community [5].

We developed a TMG model that allows us to complete an efficient perform analysis of the systems introduced in Section 2 without the need of time-consuming simulation. We present the model for the case of blocking primitives but it can be applied also to other primitives such as non-blocking ${ }^{2}$. The computation phase of a process is modeled by a single place connected to a transition whose time delay is equal to the latency of the micro-architecture implementation obtained through HLS. For the I/O phases, each channel used by the process is modeled by two places (a put-place and a get-place) that feed the same channel transition, whose time delay is equal to the minimum latency of the channel.

Example. Fig. 3 shows the portion ${ }^{3}$ of TMG associated with process $P_{2}$ in Listing 1 and Fig. 2. The serial nature of the process translates into a chain of transitions in the TMG: the transition associated to channel $a$ is followed by transition $L^{2}$ that models the computation phase of $P_{2}$ and then by three transitions, for channels $b, d$ and $f$, respectively. Each channel transition is fed by a place modeling the get (put) statement in $P_{2}$ and a place modeling the put (get) statement in the corresponding process on that channel. For instance, the transition for channel $b$ is fed by a put-place for $P_{2}$ and a get-place that is part of the model of $P_{3}$.

Since the process iteratively executes the three phases, the first read operation, e.g., get ${ }_{1}^{2}$ follows the last write operation, e.g., $p u t_{3}^{2}$. For the initial marking, a token is placed in the first get-place of each process, e.g., get $t_{1}^{2}$ to model that its behavior starts with the first read operation. Also, a token is placed on the put-place of the test-bench process ( e.g., $p u t_{1}^{s r c}$ ) to model the behavior of an environment that is always ready to provide new input data: when transition $a$ fires a new token will be available (after some latency) in $p u t_{1}^{s r c}$.

\section{CHANNEL ORDERING}

In Section 2 we explained how the system performance depends both on the micro-architecture implementing the computation phase of each process and the relative ordering of the put and get statements in their I/O phases. The graph of Fig. 4(a) represents the

\footnotetext{
${ }^{2}$ The model for the non-blocking case is given in [6].

${ }^{3}$ The complete TMG for the system is reported in [6].
} 


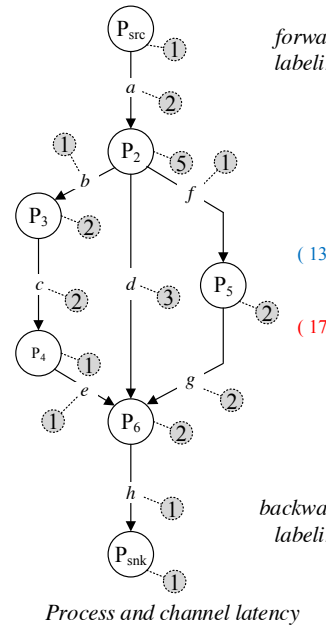

a.

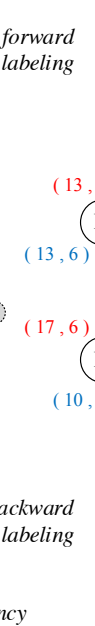

Running a

$\mathrm{P}_{4}$
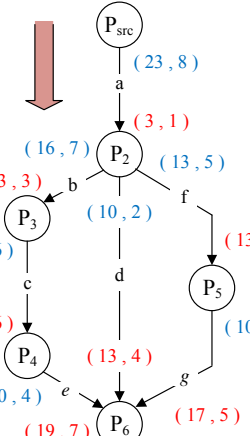

T $(2,1)$

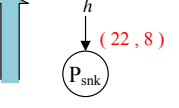

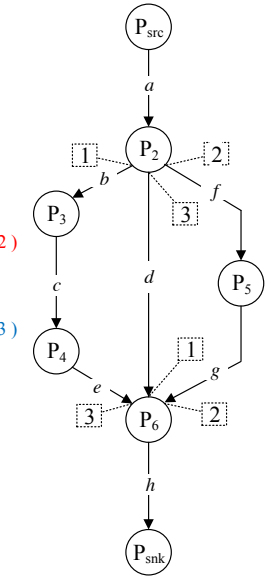

Optimal ordering c.
Fig. 4: Finding the optimal channel ordering.

result of running HLS on the system of graph Fig. 2: each vertex is now annotated with a number representing the latency of the process computation, which depends on the synthesized microarchitecture; each arc is annotated with the minimum latency required to complete the transfer of one data item on the corresponding channel ${ }^{4}$ With these latencies values, the suboptimal ordering discussed in Section 2 yields a data-processing throughput equal to 0.05 . This is the reciprocal of a cycle time equal to 20 , which can be computed with the method presented in Section 3. However, the optimum cycle time is actually 12 , i.e. $40 \%$ better. This is obtained by ordering the put statement of $P_{2}$ to access the channels as $(b, d, f)$ and the get statements of $P_{6}$ to access the channels $(d, g, e)$ (see Fig. 4(c).) Algorithm 1 finds this optimum ordering while guaranteeing also absence of deadlock.

Algorithm Description. The algorithm is a sequence of three main steps (lines 1-5): Forward Labeling, Backward Labeling and Final Ordering.

Forward Labeling performs a modified depth-first traverse of the system graph starting from the source $T_{s r c}$, while using a queue to track the vertex to be considered next (line 10-11). While visiting a vertex, each of its outgoing arcs $e=(x, y)$ is considered (line 13) following any order among its put statements (this could be an order given by the designer or the suboptimal of Section 2.) The head of arc $e$ is labeled with $(w, t)$, where $w \in \mathbb{N}^{+}$is a weight and $t \in \mathbb{N}^{+}$is a timestamp. The weight is equal to the sum of three values: the maximum among the weights associated with the arcs entering $x$, the sum of the latency of each arc leaving $x$, and the latency of $x$ (line 17). The assigned timestamp is a global progressive number for the forward traversal. Finally, if $\operatorname{arc} e$ is the last arc visiting $y$ then $y$ is put in the queue.

Example. When Forward Labeling reaches $P_{2}$, the outgoing arcs are considered in the order $f, b, d$. Hence, the corresponding timestamps are 2,3 , and 4 . The weight associated to these arcs is 13 , i.e., the sum of MaxInArcWeight $(P 2)=3$, SumOutArcLatency $(P 2)=5$, and GetVertexLatency $(P 2)=5$. See the red labels marking the arc heads in Fig. 4(b).

Backward Labeling is a similar procedure and is not reported here for lack of space ${ }^{5}$ The procedure starts from the sink vertex $T_{\text {snk }}$ and traverses the graph backward. The key difference is that when a vertex is visited, its incoming arcs are considered following the increasing order of the timestamps which have been assigned to the arc heads during Forward Labeling. Also, the weight is computed as the sum of: the maximum value of the weights associated

\footnotetext{
${ }^{4}$ A data item can be decomposed in packets to be transferred through multiple put/get iterations.

${ }^{5}$ The complete algorithm is reported in [6].
}

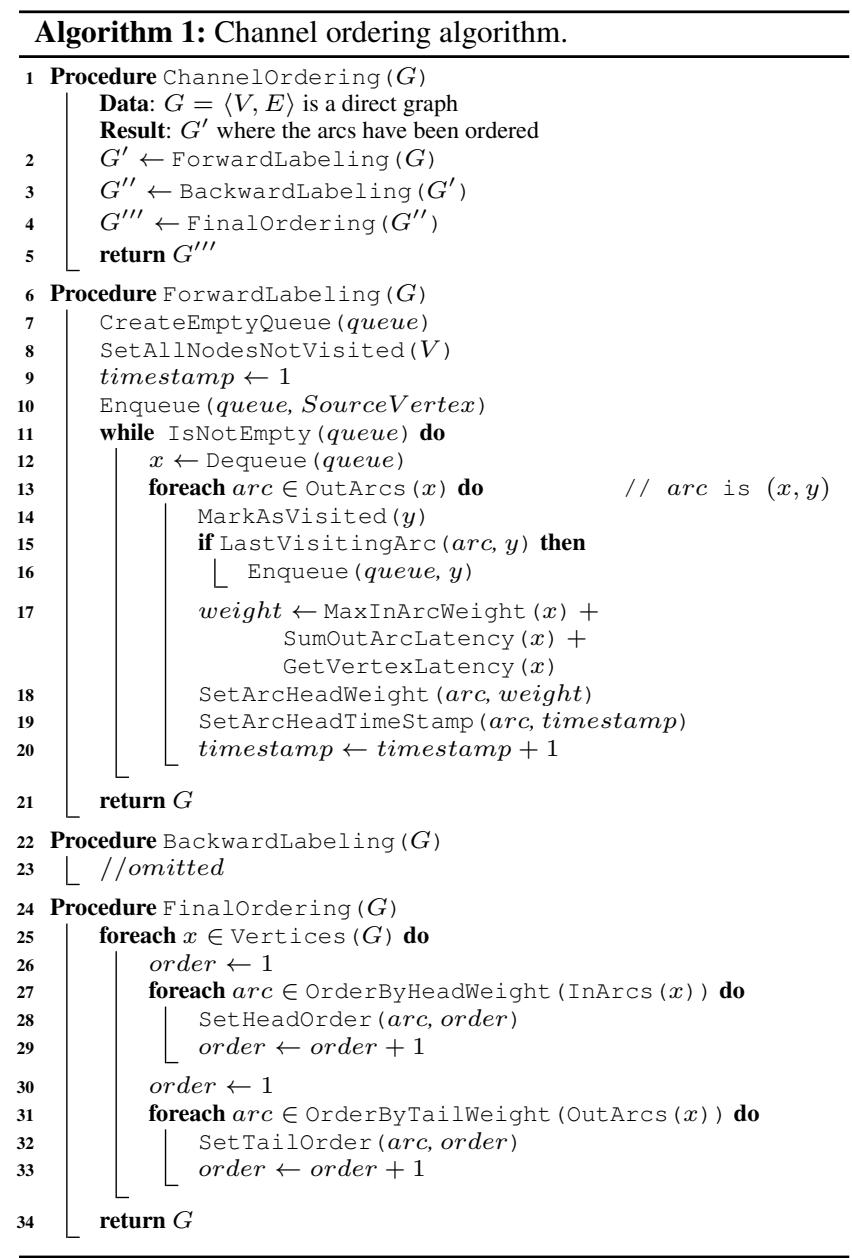

with the arcs leaving $x$, the sum of the latency of each arc entering $x$, and the latency of $x$. The assigned timestamp is a global progressive number for the backward traversal.

Example. When the backward-labeling procedure reaches $P_{6}$, its incoming arcs are considered following the order $d, g, e$. The weight associated to these arcs is 10, i.e., the sum of MaxOutArcWeight $\left(P_{6}\right)=2$, $\operatorname{SumInArcLatency}\left(P_{6}\right)=6$, and GetVertexLatency $\left(P_{6}\right)=2$. See the blue labels marking the arc tails in Fig. 4(b).

Final Ordering sorts the get statements of each process according the ascending values of the head weights (line 28) and the put statements according to descending values of the tail weights (line 32 ). In both cases, ties among the weight values are broken according the ascending values of the timestamps: this tie-break is necessary to avoid certain deadlock situations, which may occur in graphs with some symmetric structures.

Example. Since the head weights of arcs $e, d, g$ are 19,13,17, process $P_{6}$ read first from channel $d$, then $g$, and finally $e$. Also, since the tail weights of arcs $b, d, f$, are $16,10,13$, process $P_{2}$ writes first channel $b$, then $f$ and finally $d$.

The basic idea of the algorithm is to sort the chain of put statements in each process by giving priority to those statements that start a path whose aggregate latency is longer than the others and to sort the chain of get statements in each process by giving priority to those that end a path whose aggregate latency is shorter than the others. The result of this choices is an optimization of the system performance.

The overall complexity of the algorithm is $O(n \log (n))$ in the size of the graph. It requires two depth-first visits that take time $O(|E|)$ and space $O(|V|)$ in the worst case to store the verices in the queue. The sorting of the arcs takes $O(|E| \log (|E|))$, which dominates the overall complexity. 


\section{DESIGN METHODOLOGY}

In Section 4 we described how the algorithm for producing the optimal channel reordering is based on the process latencies, defined by the synthesized micro-architecture implementing their computation phase. Varying these latencies may result in a different ordering and, in turn, a different system-level performance. To explore the design space we developed the methodology based on the idea of separation of concerns [10] between computation (optimizing of process latencies) and communication (channel reordering). As shown in Fig. 5, the starting point is a system model with a set of Pareto-optimal $\mu$-architectures that differ in terms of latency and area occupation. The designer can also specify the targets (e.g. the target cycle time $T_{C T}$ ) of the overall system. At each iteration, given the current cycle time (CT) and the associated critical cycle computed with the performance model presented in Section 3, the goal is to optimize the overall system to meet the given constraints by first exploring the available $\mu$-architectures and then applying the channel ordering based on the resulting process latencies.

Given the target cycle time $T_{C T}$, we define the performance slack $s_{p}$ as $s_{p}=T_{C T}-C T$. If $s_{p}>0$, the constraint is met and we can thus perform area optimization (area recovery). If $s_{p} \leq 0$, we reduce the latency of the processes on the critical cycle (timing optimization).

Given the set of processes $P$ and the implementations $I$, a binary variable $x_{i, p} \in P \times I$ denotes if the final system includes implementation $i$ for process $p$. Each process must have one and only one implementation. The latency gain $l_{i, p}$ is the (positive or negative) difference between the current latency of process $p$ and the latency of $i$. The area gain $a_{i, p}$ is the (positive or negative) difference between the current area of $p$ and the area of $i$. These values represent the differences introduced by selecting implementation $i$ instead of the current one for process $p$.

We define two problems.

Area recovery: given a system with $s_{p}>0$, determine the IP configurations that minimize the area occupation without affecting the critical cycle (i.e., maintaining $C T<T_{C T}$ ).

This problem is a variant of the knapsack problem to maximize $\sum_{i, j} x_{i, j} * a_{i, j}$ while respecting constraint $\sum_{i, j} x_{i, j} *\left(-l_{i, j}\right) \leq$ $s_{p}$ for the processes on the critical cycle. Since the implementations are Pareto optimal, moving towards a positive area gain corresponds to a negative latency gain and vice versa.

Timing optimization: given a system with $s_{p}<0$, determine the IP configurations to minimize the difference $C T-T_{C T}$.

This problem corresponds to a classical optimization problem, where we aim at maximizing $\sum_{i, j} x_{i, j} * l_{i, j}$, i.e., the cumulative latency gain for all the processes $j$ on the critical cycle.

These problems can be formulated as instances of Integer Linear Programming (ILP), with constraints to discard the configurations already optimized. The formulation with area constraints is the dual problem and is omitted due to lack of space.

\section{EXPERIMENTAL RESULTS}

We developed ERMES, a prototype CAD tool based on the proposed methodology. It uses the GLPK (GNU Linear Programming Kit) package to solve the ILP formulations. We applied ERMES to the case study of an MPEG-2 Encoder, which had been previously designed in our team [11]. This design was refactored so that each process is specified using a loosely-timed TLM style following the common structure described in Section 2. The resulting system-level specification consists of 26 processes described in synthesizable SystemC and interconnected through 60 blocking channels (Table 1). These processes are connected to two additional processes which act as a test-bench by providing the image streams and receiving the encoded images, respectively.

The characteristics of the MPEG-2 Encoder algorithm are good to highlight the main challenges of designing a complex SoC accelerator. In particular, its system-level block diagram contains struc-

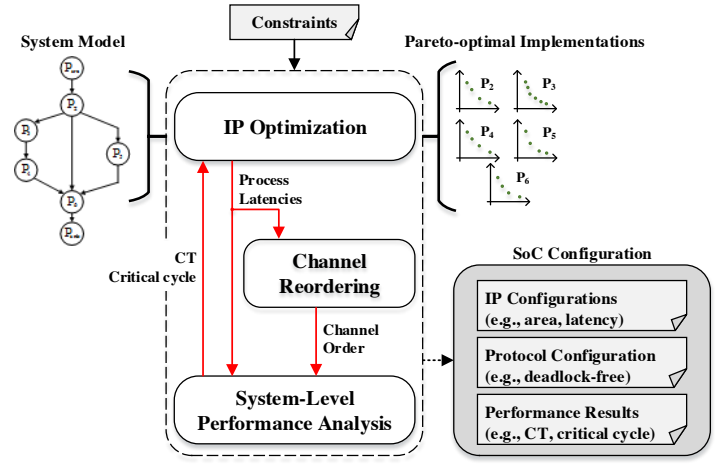

Fig. 5: Outline of the proposed methodology.

Table 1: Experimental setup of the MPEG-2 Encoder.

\begin{tabular}{l|c||l|c}
\hline Processes & 26 & Channels & 60 \\
\hline Lines of Code & $\sim 9,000$ & Image Size (pixels) & $352 x 240$ \\
\hline HLS knobs & $\begin{array}{l}\text { loop pipelining, } \\
\text { loop unrolling, .. }\end{array}$ & Pareto points & 171 \\
\hline Technology & $45 \mathrm{~nm}$ & Frequency & $1 \mathrm{GHz}$ \\
\hline
\end{tabular}

tures that may cause deadlock or performance degradation if the communication protocol is not properly configured: these include recovergent paths (as for the example of Fig. 1) and feedback loops.

By applying the method for compositional HLS and design-space exploration proposed by Liu and Carloni [11], we derived many alternative Pareto-optimal $\mu$-architectures for the inner computation core of each process. Since computation is not intertwined with communication, these characterizations are not affected by channel ordering and can be performed as a preprocessing step. We performed the characterization of the channel latencies based on the quantity of the data to be transferred and the physical constraints imposed by the HLS tool for the channels. These latencies range from 1 to 5,280 clock cycles and do not depend on channel ordering or the process implementations.

Using the approach proposed in [11], we obtained also a set of of Pareto-optimal implementations for the overall system. Notice that these implementations are optimal among all those that can be obtained without making any automatic modification to the SystemC code (including possible reordering of the interface-primitive statements within the processes.) Indeed, they are based on the choice of a conservative ordering that guarantees absence of deadlock but may introduce unnecessary serialization of processes that could run in parallel, similarly to the situation described in Section 2. Without the support of a tool like ERMES, it is difficult to go beyond such conservative ordering because there are simply too many possible ordering combinations to consider, with the additional risk of introducing a deadlock. Furthermore, the identification and correction of deadlock situation typically require multiple lengthy simulations of the entire SystemC design.

Instead, the applications of the proposed methodology and the ERMES tool allows us to perform richer design-space explorations and to obtain better implementations. To show its capabilities we report the results obtained starting from two implementations from the given set. Implementation $M 1$ has the best performance as it features the fastest implementations for the computational part of each process: specifically, it delivers a CT of 1,906 KCycles with an area occupation of $2.267 \mathrm{~mm}^{2}$. Implementation $M 2$ is the result of trading-off performance for a smaller area occupation: it has a CT of 3,597 KCycles with an area of $1.562 \mathrm{~mm}^{2}$.

When applied to implementation $M 1$, ERMES is capable of detecting some unnecessary serialization of processes that could run in parallel. By reordering the interface primitives of some processes, it resolved this issue without making any change on their core computational parts. The result is a $5 \%$ improvement of the CT without any increase in area occupation.

When applied to implementation $M 2$ with different input con- 
straints, ERMES can perform automatically two different types of iterative design-space explorations. The left-hand side of Fig. 6 shows a timing-optimization exploration as the result of imposing a constraint on the target cycle time $T_{C T}=2,000$ KCycles with the goal of substantially improving performance. The right-hand side of Fig. 6 shows an area-recovery exploration that is based on running ERMES with a much more relaxed constraint $\left(T_{C T}=4,000\right.$ KCycles) in order to reduce the area occupation. Even if they start from the same implementation $M 2$, the two explorations present a different evolution due to the different input constraints.

In the first exploration, ERMES immediately generates a new implementation that meets the target cycle time while increasing the area occupation. Then, it tries unsuccessfully to reduce the area overhead, as the second implementation violates the given constraint. The situation is recovered in the third iteration and the last one confirms that no further changes can be applied. The final implementation gives a speed-up of $2 \mathbf{x}$ with respect to the initial one, with an area overhead of $44.57 \%$.

In the second exploration the starting point already meets the target cycle time. Hence, ERMES turns to optimize the area. The first generated implementation gives a significant area reduction but violates the timing constraint. In the following iteration ERMES removes the timing violation without affecting the area occupation. After the last iteration, the resulting implementation yields an area reduction of $32.46 \%$ with respect to $M 2$, in exchange for a timing degradation of less than $1 \%$.

ERMES automatically executes very interesting optimizations. For example, during the third iteration of the first exploration, it improves the system performance by selecting much faster implementations for some of the processes on the critical cycle. The corresponding area overhead is recovered by selecting smaller implementations for other processes (including other processes on the critical cycle), provided that the cumulative balance of their latency gains remains positive. As a result, the cycle time is effectively reduced, with minor additional changes in the area occupation of the final design. Notice that, as it generates a new implementation, the algorithm for channel reordering optimizes the performance in almost all the cases, but the last ones where no changes are applied to the process latencies.

Analysis of scalability. Since the complexity of the proposed approach only depends on the system topology, we designed a set of synthetic SoC benchmarks to evaluate also its scalability. We generated graphs with up to 10,000 processes interconnected with 15,000 channels, along with a corresponding set of hypothetical $\mu$ architectures. The resulting benchmarks have characteristics similar to those of the MPEG-2, including the presence feedback loops and reconvergent paths. The experimental results demonstrate that our approach scales well, as ERMES takes a time of the order of a few minutes in the worst cases [6].

\section{RELATED WORK}

The serialization of the communication that may arise with the use of commercial HLS tools is a problem that has received limited attention in the literature. One may think that this problem can be solved by breaking the channel operations in multiple concurrent processes. However, this often leads to inefficient designs because HLS tools create as many memory ports as the number of concurrent processes insisting on that memory and the memory size scales badly with the number of ports. Many designers thus prefer to reduce the number of processes and use a design style with three phases (input reading, computation and output writing) like the one assumed in Section 2. This simplifies system-level analysis and optimization and has been effectively adopted in many works based on synchronous dataflow and Kahn process networks (e.g. [9, 19]). These models of computation, however, lead to communication channels based on FIFOs, which must be carefully sized [10]. Also, they do not handle the serialization issues introduced by the inter-
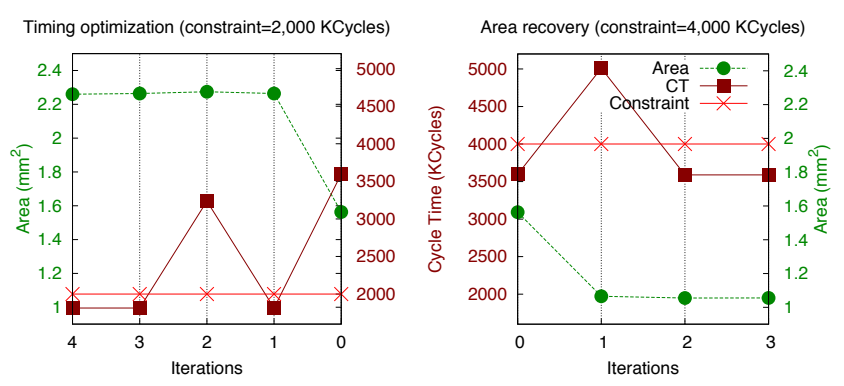

Fig. 6: Design-space exploration starting from $M 2$.

face primitives offered with modern HLS tools. Deadlock detection has been studied for blocking primitives in CSP systems [17], but the interactions and the ordering among the channels was not considered. Deadlock removal has been addressed in the context of SystemC simulation kernels [1], but no approaches were proposed for preventing a deadlock and optimizing the system performance in hardware designs described in SystemC.

\section{CONCLUSIONS}

We proposed a novel methodology and an associated CAD tool for supporting compositional HLS of complex SoC accelerators. It focuses on the integration of IP components through the use of the interface libraries offered by HLS commercial tools and cooptimizes the computation micro-architecture and the communication channels. The experimental results show the efficiency of our approach in the automatic optimization of a complex design. Future work will involve the co-optimization of the memory elements.

Acknowledgments. This work is partially supported by the STARnet Center for Future Architectures Research (C-FAR), the DARPA PERFECT program, and the NSF (A\#:1219001).

\section{REFERENCES}

[1] C.-N. Chou et al. Formal deadlock checking on high-level systemc designs. In Proc. of ICCAD, pages 794-799, 2010.

[2] J. Cochet-Terrasson et al. Numerical computation of spectral elements in max-plus algebra. In Proceedings of IFAC CSSC, 1998.

[3] F. Commoner, A. W. Holt, S. Even, and A. Pnueli. Marked directed graphs. Journal of Computer and System Sciences, 5(5):511-523, 1971.

[4] P. Coussy and A. Morawiec. High-level synthesis: from algorithm to digital circuit. Springer, 2008.

[5] A. Dasdan, S. Irani, and R. K. Gupta. An experimental study of minimum mean cycle algorithms. Technical Report 98-32, UC Irvine, 1998.

[6] G. Di Guglielmo, C. Pilato, and L. P. Carloni. A design methodology for compositional high-level synthesis of communication-centric SoCs. Technical report, Dept. of Computer Science, Columbia University, New York, Apr. 2014.

[7] M. Fingeroff. High-level synthesis blue book. Mentor Graphics Corp., 2010.

[8] F. Ghenassia. Transaction-Level Modeling with SystemC. Springer-Verlag, 2006.

[9] K. Huang et al. Embedding formal performance analysis into the design cycle of mpsocs for real-time streaming applications. ACM Trans. Embed. Comput. Syst., 11(1):8:1-8:23, 2012.

[10] K. Keutzer, A. R. Newton, J. M. Rabaey, and A. Sangiovanni-Vincentelli. System-level design: Orthogonalization of concerns and platform-based design. Trans. Comp.-Aided Des. Integ. Cir. Sys., 19(12):1523-1543, 2006.

[11] H.-Y. Liu, M. Petracca, and L. P. Carloni. Compositional system-level design exploration with planning of high-level synthesis. In Proc. of DATE, pages 641-646, 2012.

[12] J. Magott. Performance evaluation of concurrent systems using petri nets. Information Processing Letters, 18(1):7-13, 1984.

[13] G. Martin and G. Smith. High-level synthesis: Past, present, and future. IEEE Design \& Test, 26(4):18-25, 2009.

[14] T. Murata. Petri nets: Properties, analysis and applications. Proc. of the IEEE, 77(4):541-580, 1989.

[15] Open SystemC iv Initiative (OSCI). OSCI TLM-2.0 Language Reference Manual. 2009.

[16] J. Sanguinetti, M. Meredith, and S. Dart. Transaction-accurate interface scheduling in high-level synthesis. In ESLsyn Conference, pages 31-36, 2012.

[17] B. Shao, N. Vasudevan, and S. A. Edwards. Compositional deadlock detection for rendezvous communication. In Proc. of EMSOFT, pages 59-66, 2009.

[18] M. Taylor. Is dark silicon useful? Harnessing the four horsemen of the coming dark silicon apocalypse. In Proc. of DAC, pages 1131-1136, June 2012.

[19] S. van Haastregt and B. Kienhuis. Automated synthesis of streaming C applications to process networks in hardware. In Proc. of DATE, pages 890-893, 2009. 